\title{
Portable multispectral imaging system based on light-emitting diodes for spectral recovery from 370 to $1630 \mathrm{~nm}$
}

\author{
Jorge Herrera-Ramírez, ${ }^{*}$ Meritxell Vilaseca, and Jaume Pujol \\ Centre for Sensors, Instruments and Systems Development (CD6), \\ Universitat Politècnica de Catalunya (UPC), Terrassa, Barcelona, Spain \\ ${ }^{*}$ Corresponding author: jorge.alexis.herrera @ cd6.upc.edu
}

Received 13 February 2014; revised 12 April 2014; accepted 14 April 2014; posted 15 April 2014 (Doc. ID 206391); published 9 May 2014

\begin{abstract}
To expand and investigate the potential of spectral imaging, we developed a portable multispectral system using light-emitting diodes. This system recovers spectral information from the UV to the near IR over a large area using two different image sensors synchronized with 23 bands of illumination. The system was assessed for spectral reconstruction through simulations and experimental measurements by means of two methods of spectral reconstruction and three different evaluation metrics. The results over a Macbeth ColorChecker chart and other samples, including pigments usually employed in art paintings, are compared and discussed. The portable multispectral system using LEDs constitutes a cost-effective and versatile method for spectral imaging. (C) 2014 Optical Society of America

OCIS codes: (110.4234) Multispectral and hyperspectral imaging; (230.3670) Light-emitting diodes; (110.0110) Imaging systems; (120.4820) Optical systems.

http://dx.doi.org/10.1364/AO.53.003131
\end{abstract}

\section{Introduction}

Spectral imaging is a field of research with many scientific and technological applications such as remote sensing [1], color imaging [2], biometrics and medicine [ $[3-7]$, cultural heritage and art work studies $[\underline{8}-10]$, and the food industry $[\underline{11}, 12]$. Different multispectral imaging systems have been devised to encompass this wide range of applications, mainly single point spectrometers with $2 \mathrm{D}$ scanning systems [13], digital cameras combined with line-scan spectrographs [12], color filter wheels [14], filter mosaics $[15]$, lenslet arrays and narrowband filters $[\underline{6}, \underline{16}]$, and tunable filters of liquid crystal or acousto-optic technology $[17,18]$. Each of these systems has compromises with regard to operational speed, spectral and spatial resolutions, and cost. For instance, 2D scanning, line scanning, and filter wheel systems

$1559-128 \mathrm{X} / 14 / 143131-11 \$ 15.00 / 0$

(C) 2014 Optical Society of America usually take a long time to acquire the spectral data. In contrast, digital camera systems combined with lenslet arrays or filter mosaics overcome this shortcoming with snapshot acquisitions, though at the cost of spectral or spatial resolution or both. In the case of tunable filters, they can handle fast changes in wavelength with good spectral and spatial resolution, but they are more costly and present limitations related to the field-of-view (FOV) and spectral purity in some of their bands. It can be said that the suitability of a multispectral system has to be determined by evaluating the specific requirements of the application and the characteristics of the different technologies.

To acquire multispectral imaging data, all these conventional approaches require a white light source such as a halogen or mercury-xenon lamp. However, the current development and availability of the lightemitting diode (LED) technology provide new solutions for setting up a multispectral system. LED illumination technology is economical and efficient, 
has a long life cycle, and is constantly evolving. LEDs have a narrow spectral emission and are available in several wavelengths over the different spectral ranges of the ultraviolet (UV), the visible (VIS), and the near-infrared (NIR). They also enable illumination with a wide number of different wavelengths or customized combinations of wavelengths speedily and in switching synchrony with the imaging sensors. They can also be combined with filters to control the spectral emission of the LEDs or to evaluate luminescence [4].

Seeking to take advantage of the properties provided by the LED elements, some proposals of multispectral systems using LED illumination and covering the VIS range of the electromagnetic spectrum have been recently published $[3, \underline{4}, 19-22]$. For example, Bouchard et al. [4] proposed a low-cost two-channel system with active LED illumination combined with filters in the visible region for fast acquisition of cortical oxygenation, blood flow, and intracellular calcium dynamics. They obtained frame rates of over $220 \mathrm{~Hz}$, allowing two wavelength spectral images to be acquired exceeding $110 \mathrm{~Hz}$. Everdell and colleagues [3] developed an LED based system used for fast ocular fundus imaging to avoid issues of image shifting due to the natural movements of the eye. Again in this case, the versatility for selecting specific wavelengths and the capacity of fast strobe using low-cost elements became major advantages for the application. In 2012, Fauch and co-workers [21] presented a study of parameters that affect the accuracy of reflectance spectrum recovery from the compressed data obtained with a system that consisted of a computer-controlled set of LEDs coupled to a thick fiber. They showed that the performance of the system is better when the spatial uniformity of the object illumination is increased. In other words, they showed that LED light sources must be carefully designed taking this fact into account. Finally, Li and Chan [22] developed a reflection hyperspectral imaging system covering the 350-1000 nm spectral range that comprised a UVVIS-NIR Fourier transform imaging benchtop spectrometer. This system, composed of a $\mathrm{He}-\mathrm{Ne}$ laser and LEDs, was successfully used for microspectroscopic measurements in molecular multiplex assays. The main advantages of this system are the capacity of adjustable spectral resolution and broad spectral range. From these examples it is clear that LEDs offer the characteristics of reduction of complexity of the systems, versatility in the implementation for specific applications, and fast acquisition rates and typically allow low-cost implementation and modifications.

In this study, we developed a portable multispectral system based on LED illumination covering a wide spectral range and using off-the-shelf components. The proposed system is intended for exploring and broadening the possibilities of LEDs to the near UV (nUV) and NIR spectral regions, specifically from 370 to $1630 \mathrm{~nm}$. The access to this range is a desirable feature of multispectral systems, particularly in art work studies, where the most robust analysis and identification of artists' materials require spectral data from 350 to $2500 \mathrm{~nm}$ [23], or in the food industry, in which the NIR and shortwave IR (SWIR) regions can be successfully used to predict quality attributes [12]. We analyzed the performance of this system in reconstructing spectral information through computational simulations, assuming real noise conditions and also carrying out experimental measurements. The spectral reconstructions were obtained using the pseudoinverse method for spectral estimation $[14,24]$ and the spline interpolation technique $[25,26]$. To quantitatively compare and evaluate the results, we used three different metrics: the color difference formula CIEDE2000 (DE00) [27-29], the goodness-of-fit coefficient (GFC) [30,31], and the root mean square error (RMSE) [14,31]. The results showed good overall system performance. Better results were obtained in the nUV and VIS than in the NIR region because of the differences in the sensing camera used and the LED elements' density in this last region. This novel portable system prototype provides a simple, versatile, and cost-effective solution suitable for projects focused on spectral imaging.

\section{Experimental Setup}

\section{A. Equipment}

The proposed system, shown in Fig. 1, consists of two modules. Module 1 comprises a cooled CCD monochrome camera (Qimaging QICAM with $1392 \times$ 1040 pixels of sensitive area and 12 bit depth of

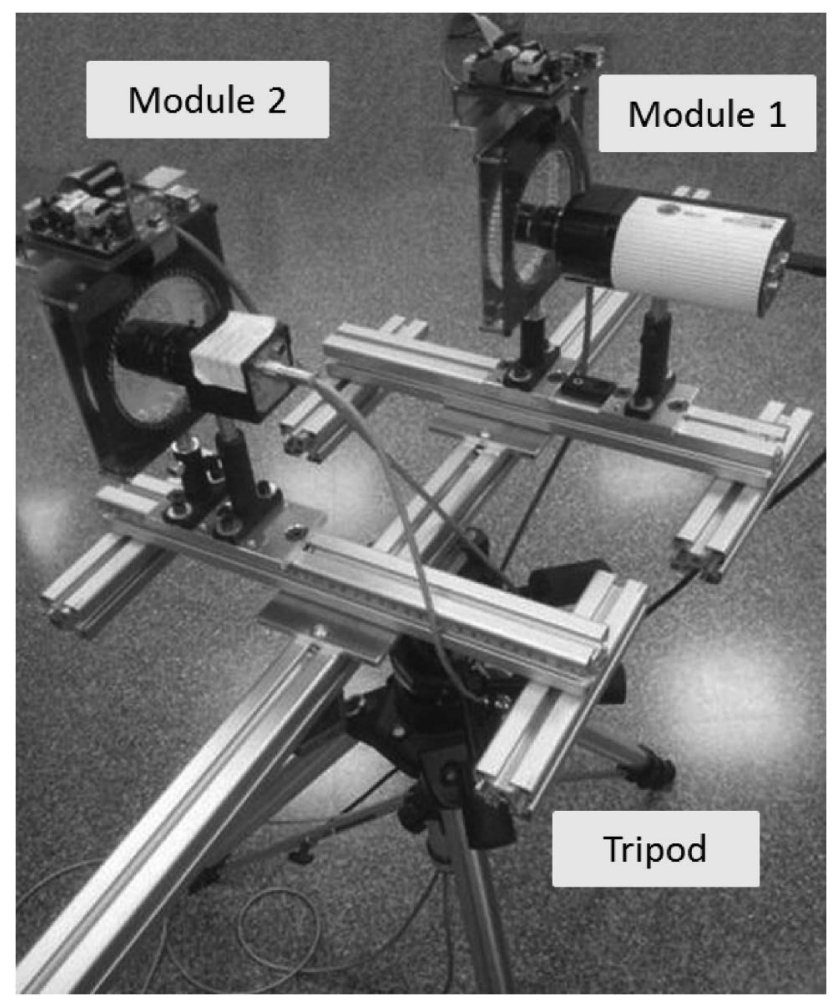

Fig. 1. Portable multispectral system setup. 
digitalization) with a sensitivity over the range of $370-950 \mathrm{~nm}$ and a set of LEDs with 16 different emission wavelengths that match the spectral sensitivity of the camera (peak wavelengths of emission in nanometers: $373,404,432,461,500,353,593,634$, $665,693,728,761,801,835,874$, and 903). Module 2 has an InGaAs camera with a sensitivity from 930 to $1630 \mathrm{~nm}$ and a set of LEDs with seven different emission wavelengths over this spectral range (peak wavelengths of emission in nanometers: 955, 1071, $1202,1297,1451,1540$, and 1630); this InGaAs camera from Hamamatsu has a sensitive area of $340 \times$ 256 pixels and 14 bit depth of digitalization. In total, the proposed multispectral system covers the spectral range from 370 to $1630 \mathrm{~nm}$ with 23 channels provided by the LED illumination.

Regarding the optics, a lens (Cinegon 1.4/12 mm Series Compact) with high transmittance in the $400-1000 \mathrm{~nm}$ region is used in module 1 , whereas a lens (Kowa LM12HC-SW 1.4/12.5 mm SWIR) with improved transmittance between 800 and $2000 \mathrm{~nm}$ is used in module 2.

Figure 2 shows the emission spectra of LEDs separated by their respective modules. Their full width at half-maximum (FWHM) ranges from 9.5 to $45 \mathrm{~nm}$ for the constituents of module 1 and from 51 to $126 \mathrm{~nm}$ for the constituents of module 2 . The separation between emission peaks is around $40 \mathrm{~nm}$ and above $90 \mathrm{~nm}$ in modules 1 and 2 , respectively. This increased separation in module 2 is caused by the reduced commercial availability of LED elements in the NIR.

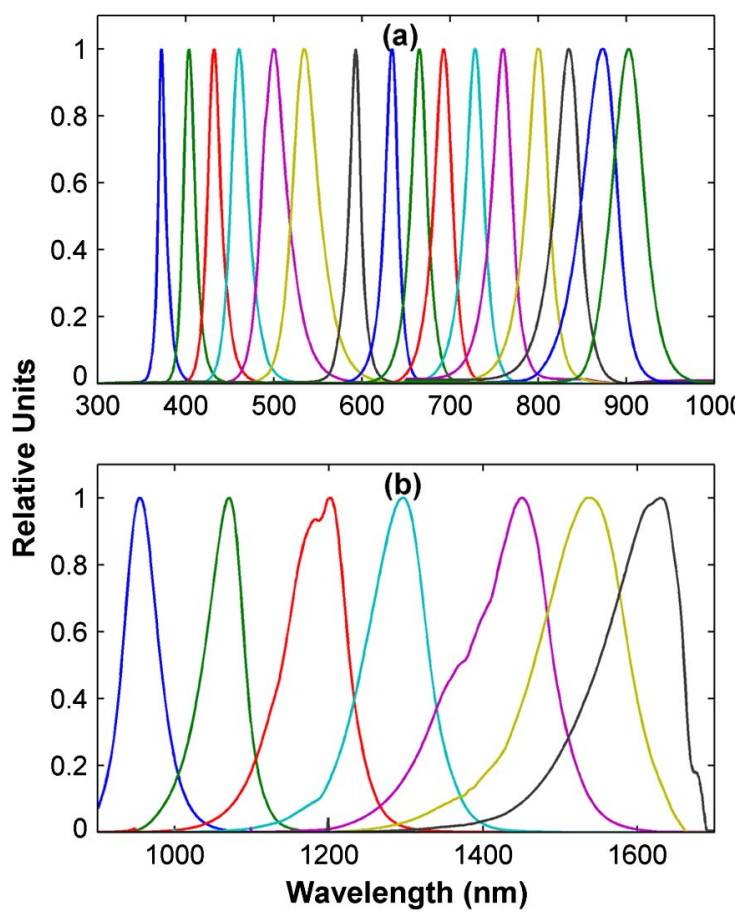

Fig. 2. Emission spectra of LEDs. (a) of module 1 and (b) of module 2. Measurements were carried out with commercial scanning spectrometer model Spectro 320 R5 of Instrument Systems.

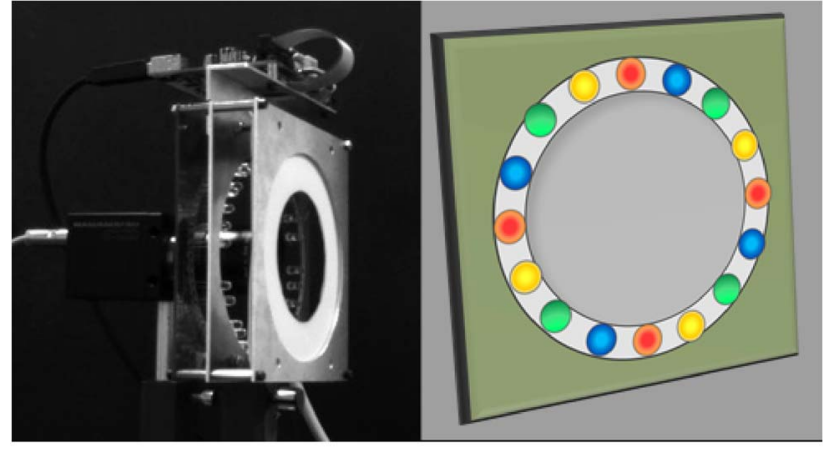

(a)

(b)

Fig. 3. Distribution of the LEDs in the illumination module (a) picture of one of the modules of the prototype and (b) illustrative sketch of the distribution of the LEDs according to their emission wavelength.

Figure 3(a) shows the prototype of one illumination module together with its corresponding camera. The LED elements are spatially distributed over a ring that allows the cameras to capture the images of the samples through its hole. Figure 3(b) shows a simplified schematic layout of the location of the LEDs corresponding to the same peak wavelength of emission. The LEDs are distributed with an angular separation of $90^{\circ}$, invoking a symmetry criterion already used by other authors [20]. Other options using angles like $45^{\circ}$ have been described in the bibliography of [32], although they have not been considered here due to the large number of wavelengths in our system setup. With this spatial arrangement and using a diffuser filter in front of the LED elements, a diffuse and rather uniform illumination on the sample was obtained. Therefore, the system uses geometry of diffuse illumination and observation at $0^{\circ}\left(\mathrm{d} / 0^{\circ}\right)$

A customized computational interface was developed to control all parameters of the system. From this same interface we perform the synchronization of image acquisition and LED illumination, noise correction, calibration, and processing of acquired information. The system has been designed in a simple modular way that can be adapted to different applications, so that based on any specific configuration (camera apertures, working distances, requirements in speed of acquisition, and FOV), different spatial resolutions and times of exposure could be worked out.

\section{B. Collection of nUV-VIS-NIR Image Cubes}

Spectral image cubes for the whole proposed range are obtained in sequence using the two cameras described. The system is used in dark conditions to control other sources of light that could have effects on the sample while acquiring the images. To control the influence of temporal sources of noise, i.e., of the illumination and the sensors, each image of the cube is the result of averaging 10 frames captured after the emission of the LED illumination has stabilized during a particular time [33,34]. The stabilization 
times are predetermined for each channel to ensure that the emission does not change more than $1 \%$ during the frames' acquisition. These times range between 1 and $5 \mathrm{~s}$ depending on the channel.

To correct the spatial nonuniformity for the response of the pixels of the camera and the illumination, a flat fielding process based on capturing images of dark frames and of a calibrated white standard panel (Gigahertz-Optik BN-R98-SQ12, 98\% reflective) is carried out. This procedure was proposed by de Lasarte et al. [35] and consists of calculating gain and offset matrices to correct individual fluctuations on every pixel. The dark and white standard images are captured under specific camera parameters of offset, gain, and exposure time for each channel, which are also later used to collect the images from the samples. The use of the white standard allows the process of calibration of the exposure times to exploit the maximum of the dynamic range of the cameras. This white standard also helps to assimilate spectral images as reflectance factor images after proper calibration. In this study we established the suitable parameters for the system FOV to fit the surface size of the white standard panel used in this calibration, i.e., $25 \mathrm{~cm} \times 25 \mathrm{~cm}$. Thus, the spatial resolutions are approximately $180 \mu \mathrm{m}$ in module 1 and $735 \mu \mathrm{m}$ in module 2. Also for this configuration, the acquisition of the spectral images, after calibration, takes a total time of $94 \mathrm{~s}$.

\section{Principle of Operation}

\section{A. Recovery of Spectral Reflectances}

The camera responses, $X_{i}$, in arbitrary units of an imaging system when an object with reflectance $R(\lambda)$ is under a specific illumination $I_{i}(\lambda)$ can be expressed as

$$
X_{i}=\int_{\lambda} I_{i}(\lambda) R(\lambda) S(\lambda) \mathrm{d} \lambda,
$$

where $S(\lambda)$ is the spectral sensitivity of the camera sensor. Assuming a discretely sampled description of the former quantities without considerable loss of information, Eq. (1) can be rewritten in a matrix way as follows:

$$
\mathbf{x}=\mathbf{C}^{T} \mathbf{r},
$$

where $\mathbf{r}$ is a reflectance column vector at $p$ sampling wavelengths, $\mathbf{x}$ is a $q$-component column vector of the camera responses, and $\mathbf{C}$ is a $p \times q$ matrix that contains the product of the spectral sensitivity of the camera and the spectral emission of $q$ different LED illuminations.

The key question in a multispectral system is to recover the spectral reflectance $\mathbf{r}$ of a sample given a vector of camera responses $\mathbf{x}$. Several methods have been proposed to this end [24,36]. In this study, we used the well-known spline interpolation method
$[25,26]$ and the pseudoinverse method with training $[\overline{14}, \overline{24}]$ for spectral estimation.

The interpolation methods consider the entries of the vector $\mathbf{x}$, after proper calibration with a standard white or a sample with known spectral reflectance, as the responses to a sampling in specific wavelengths of the reflectance curve. The information in these wavelengths is then interpolated to recover the complete spectral reflectance, without requiring prior knowledge of the type of reflectances to be interpolated or the spectral characteristics of the system. The accuracy of the interpolation methods is greatly dependent on the separation of the sampling, or equivalently, the number of spectral channels. Therefore, the system can be considered as a spectrometer with low resolution. In this study, the spline interpolation method was used on account of its characteristic smoothness suited to the expected type of spectral functions for real materials.

The solution that the pseudoinverse model for spectral estimation provides is a matrix that operates by mapping camera responses to estimated reflectances. This mapping matrix minimizes the least-squares error for a training set of known reflectances with the corresponding camera responses. It is therefore assumed that the training set is a good representation of the test set, i.e., unknown reflectance curves that will be later measured with the system. If we let $\mathbf{R}_{t}$ be a matrix of $m$ columns of training reflectance samples and $\mathbf{X}_{t}$ a matrix of $m$ columns of system responses to the training set, then the matrix D that takes $\mathbf{X}_{t}$ to $\mathbf{R}_{t}$ is given by

$$
\mathbf{D}=\mathbf{R}_{t} \mathbf{X}_{t}^{T}\left(\mathbf{X}_{t} \mathbf{X}_{t}^{T}\right)^{-1},
$$

where $\mathbf{X}_{t}^{T}\left(\mathbf{X}_{t} \mathbf{X}_{t}^{T}\right)^{-1}$ is the so called Moore-Penrose pseudoinverse of the matrix $\mathbf{X}_{t}$. By applying a matrix $\mathbf{D}$ to a system response vector $\mathbf{x}$, i.e., $\mathbf{r}=\mathbf{D x}$, a reflectance vector $\mathbf{r}$ is estimated.

\section{B. Performance Evaluation}

Several metrics to evaluate the quality of spectral estimations in multispectral systems are available [31], each with specific characteristics suited to different purposes (major colorimetric closeness or spectral fitting). In this study we implemented three different metrics to evaluate the system's performance. Two metrics were used to compare the estimated spectral curves in relation to the original spectra: the RMSE [14,31], a widely used metric for spectral evaluation; and the GFC proposed by Hernández-Andrés et al. [30,31]. The GFC is based on the Schwartz inequality and is described as follows:

$$
\mathrm{GFC}=\frac{\left|\sum_{j} r_{o}\left(\lambda_{j}\right) r\left(\lambda_{j}\right)\right|}{\left\{\sum_{j}\left[r_{o}\left(\lambda_{j}\right)\right]^{2} \sum_{j}\left[r\left(\lambda_{j}\right)\right]^{2}\right\}^{1 / 2}},
$$

where $r_{o}\left(\lambda_{j}\right)$ is the original spectral data at the wavelength $\lambda_{j}$ and $r\left(\lambda_{j}\right)$ is the estimated spectrum at the 
wavelength $\lambda_{j} . \quad$ GFC $\geq 0.995, \quad$ GFC $\geq 0.999$, and GFC $\geq 0.9999$ are required for acceptable, good, and excellent matches, respectively. The third metric is DE00 [27-29] used over the reconstructions in the VIS range as a colorimetric evaluation. To compute the color data, we used the CIE D65 illuminant and CIE $196410^{\circ}$ standard observer discretized at $5 \mathrm{~nm}$ wavelength intervals.

\section{Results and Discussion}

\section{A. Simulated Results}

A preliminary analysis of the system's performance was carried out by means of simulations. To this end, the sensitivities provided by the manufacturers of the cameras and the spectral emissions of the LEDs measured in their characterization were taken into account. The spectral curves of the classic Macbeth ColorChecker (CCCR) chart consisting of 24 color patches were used as the training and test set of samples. Figure $\underline{4}$ shows the spectral curves for the spectral ranges corresponding to modules 1 and 2 . They are presented in four graphs to help the visualization: 12 spectra in Figs. 4(a) and 4(b) and the other 12 spectra in Figs. 4(c) and 4(d). In the case of the pseudoinverse method, the samples of the CCCR were used for both the training and test sets. The simulated results were obtained for three different conditions: the system under ideal conditions, under the influence of quantization error noise (i.e., with 12 bit and 14 bit noise for modules 1 and 2 , respectively), and finally under the combination of quantization error and additive random sources of noise (2\%). Realistic random noise levels around $2 \%$ have been suggested for cameras in some recent measurements [37,38].

Figure $\underline{5}$ shows the reflectance curves of the cyan color patch under the three different conditions of simulation. The results for the ideal and quantization noise conditions are in a substantial level of agreement with the real curve in all graphs in Fig. 5, since they overlap over a considerable part of the spectral range. The curves obtained for the additive random and quantization noises are less close to the real curve, though a fair level of agreement can still be observed. Comparing the methods of spectral reconstruction, the pseudoinverse technique [Figs. 5(a) and 5(b)] provides better results than the spline interpolation [Figs. 5(c) and 5(d)]; with regard to the modules of the system, module 1 [Figs. 5(a) and 5(c)] performs better than module 2 [Figs. $5(\bar{b})$ and $5(\overline{\mathrm{d}})]$.

Tables 1,2 , and 3 quantitatively summarize the simulated results for the set of CCCR color patches. Table 1 shows the main statistical descriptors of the evaluation metrics for the simulations, assuming ideal conditions of acquisition. These metrics are excellent for the pseudoinverse method of reconstruction in modules 1 and 2 . In the case of the spline interpolation method, the results show a less accurate performance, an expected outcome since this method, unlike the pseudoinverse, does not include prior information of the samples' spectral curves and assumes the responses as reflectances at discrete wavelengths. The interpolation method shows a slightly better performance for module 2 than for module 1. Despite the larger density of spectral

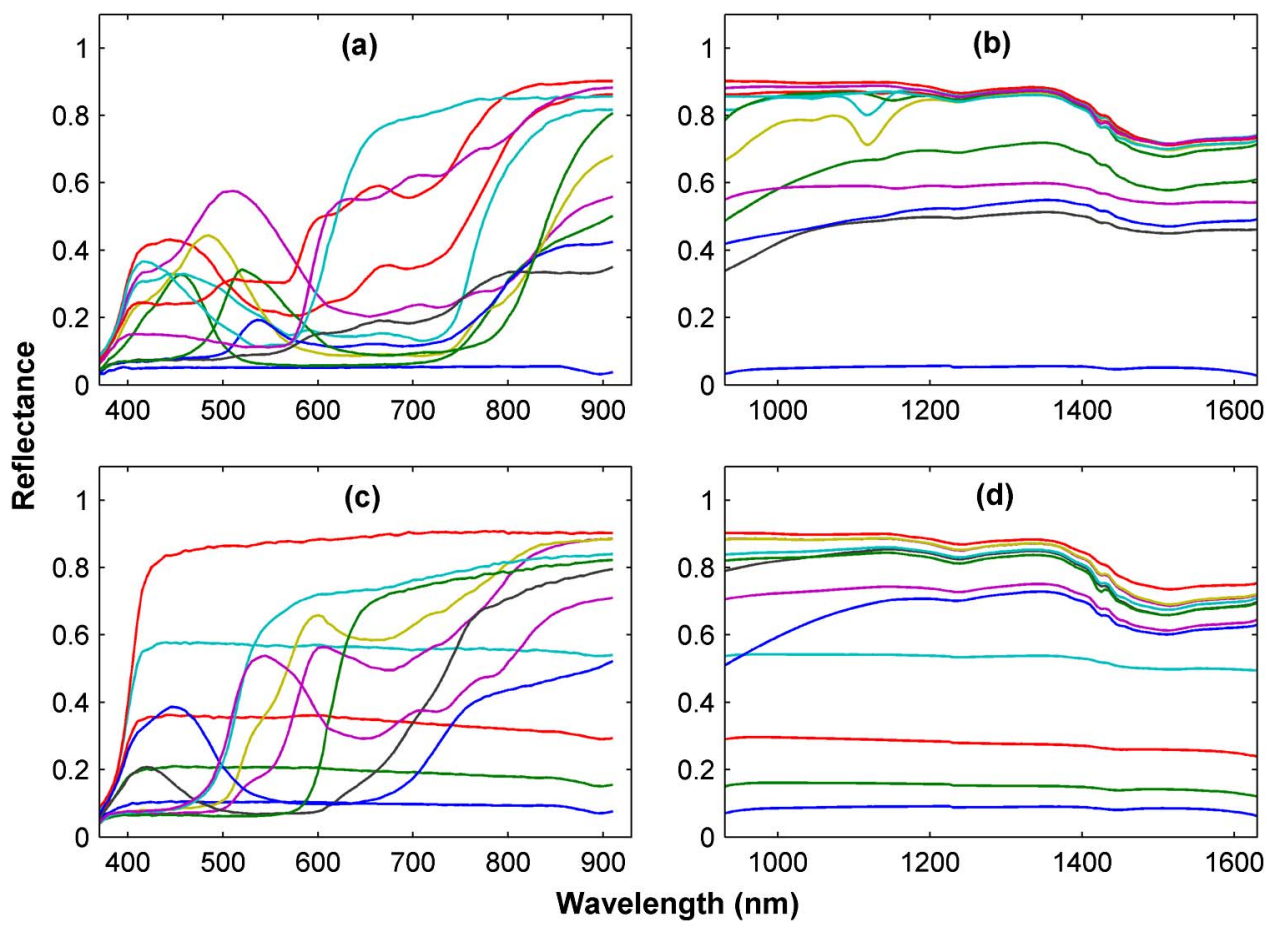

Fig. 4. Spectral curves of the classic Macbeth ColorChecker (CCCR) divided into two groups of 12 samples (a), (c) the spectral range of module 1 , (b), (d) the spectral range of module 2 . The curves were measured with commercial scanning spectrometer model Spectro 320 R5 of Instrument Systems. 


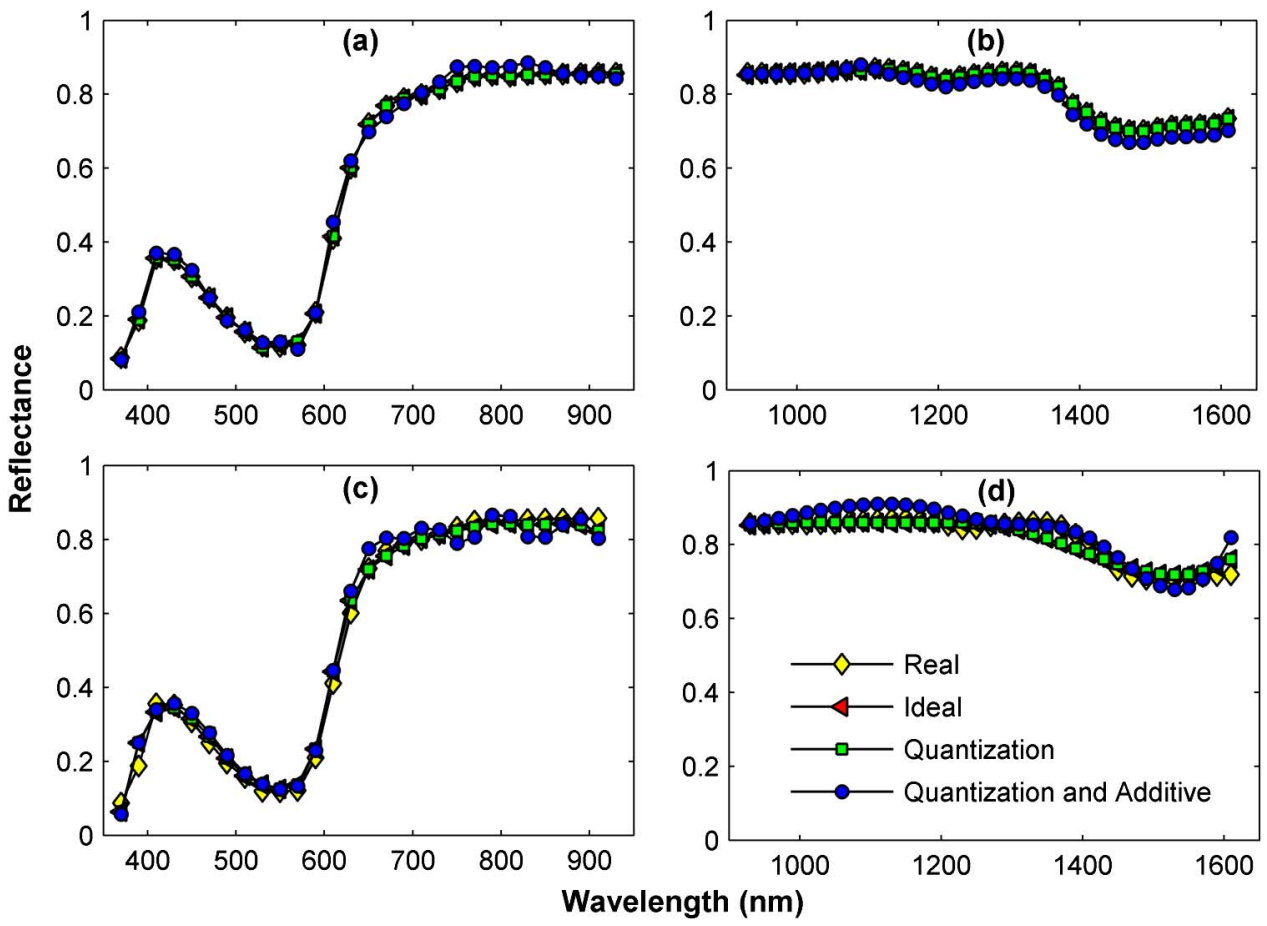

Fig. 5. Spectral reflectances of the cyan color patch in simulations under ideal, quantization, and joint additive random and quantization noise conditions. (a) Results for module 1 using the pseudoinverse reconstruction method, (b) results for module 2 using the pseudoinverse reconstruction method, (c) results for module 1 using the spline interpolation method, and (d) results for module 2 using the spline interpolation method. Real curves were obtained with commercial scanning spectrometer model Spectro 320 R5 of Instrument Systems. Ideal curves are not visible, since they are exactly located under the quantization ones.

Table 1. Evaluation Metric Results in Simulations under Ideal Conditions for the CCCR Color Patches ${ }^{a}$

\begin{tabular}{|c|c|c|c|c|c|c|c|c|c|c|}
\hline & \multicolumn{6}{|c|}{ Module 1} & \multicolumn{4}{|c|}{ Module 2} \\
\hline & PSE & Interp. & PSE & Interp. & PSE & Interp. & PSE & Interp. & PSE & Interp. \\
\hline Mean & 0.150 & 1.391 & 0.208 & 1.947 & 1.0000 & 0.9991 & 0.189 & 1.607 & 0.9999 & 0.9997 \\
\hline Min & 0.026 & 0.013 & 0.086 & 0.214 & 0.9997 & 0.9969 & 0.039 & 0.213 & 0.9979 & 0.9991 \\
\hline Max & 0.402 & 3.345 & 0.371 & 3.689 & 1.0000 & 0.9998 & 0.732 & 2.686 & 1.0000 & 1.0000 \\
\hline St. Dev. & 0.105 & 0.938 & 0.095 & 0.902 & $5.5 e-5$ & $6.96 e-4$ & 0.171 & 0.844 & $4.19 e-4$ & $1.90 \mathrm{e}-4$ \\
\hline
\end{tabular}

${ }^{a}$ PSE, pseudoinverse; Interp., spline interpolation.

bands in module 1 , this result could be attributed to the shape of the spectral curves in the respective spectral ranges of each module. The spectral curves are smoother and more similar in the range of module 2, as seen in Figs. 4(c) and 4(d), where the influence of the substrate material is evident.
Table 2 contains the main statistical descriptors of the evaluation metrics for the simulations under the influence of quantization noise. These results are almost identical to the results in Table 1 , indicating that the quantization error has a negligible effect on the spectral reconstructions when using 12 bit and

Table 2. Evaluation Metric Results in Simulations under Quantization Noise Influence for the CCCR Color Patches ${ }^{a}$

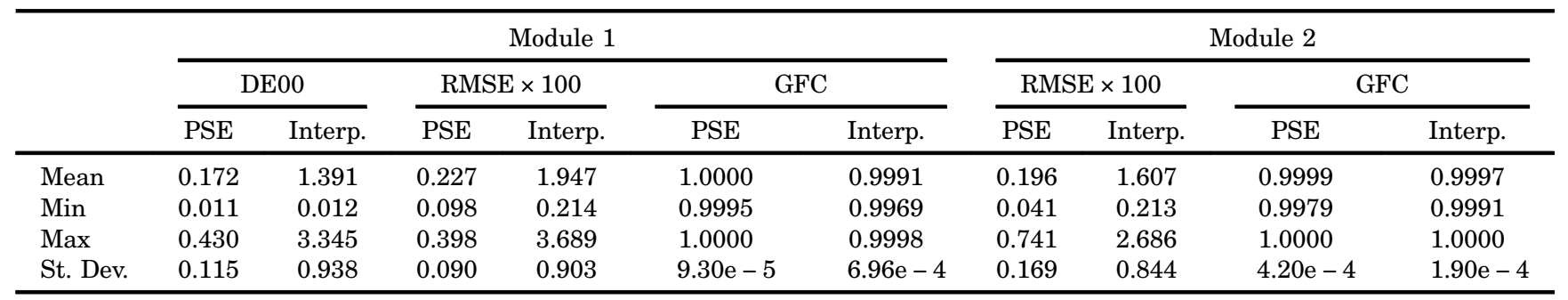

${ }^{a}$ PSE, pseudoinverse; Interp., spline interpolation. 


\begin{tabular}{|c|c|c|c|c|c|c|c|c|c|c|}
\hline & \multicolumn{6}{|c|}{ Module 1} & \multicolumn{4}{|c|}{ Module 2} \\
\hline & \multicolumn{2}{|c|}{ DE00 } & \multicolumn{2}{|c|}{ RMSE $\times 100$} & \multicolumn{2}{|c|}{ GFC } & \multicolumn{2}{|c|}{ RMSE $\times 100$} & \multicolumn{2}{|c|}{ GFC } \\
\hline & PSE & Interp. & PSE & Interp. & PSE & Interp. & PSE & Interp. & PSE & Interp. \\
\hline Mean & 1.003 & 2.366 & 1.286 & 2.245 & 0.9996 & 0.9986 & 1.90 & 3.543 & 0.9987 & 0.9984 \\
\hline Min & 0.121 & 0.811 & 0.260 & 0.297 & 0.9988 & 0.9962 & 0.67 & 0.375 & 0.9832 & 0.9964 \\
\hline Max & 3.290 & 5.719 & 2.212 & 3.709 & 0.9999 & 0.9996 & 3.31 & 6.128 & 1.0000 & 0.9993 \\
\hline St. Dev. & 0.702 & 1.239 & 0.599 & 0.959 & $3.13 e-4$ & $6.64 \mathrm{e}-4$ & 0.76 & 1.537 & $3.46 e-3$ & $7.73 e-4$ \\
\hline
\end{tabular}

${ }^{a}$ PSE, pseudoinverse; Interp., spline interpolation.

14 bit depth cameras. Consequently, in Fig. $\underline{5}$ ideal and quantization curves are always overlapped. However, a slight decrease in performance over the results of the pseudoinverse method is observed, an indication of the effect of any source of noise on this reconstruction method. Still, these results

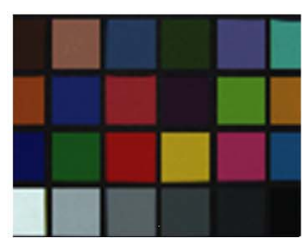

RGB

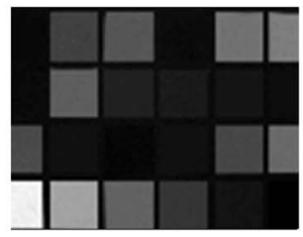

$461 \mathrm{~nm}$

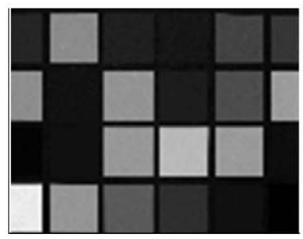

634nm

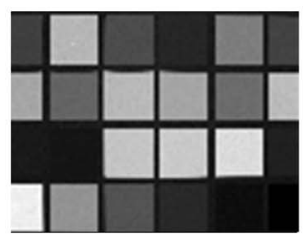

$761 n m$

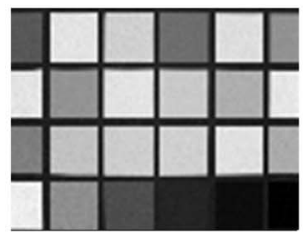

$903 n m$

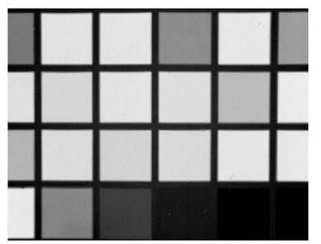

$1297 \mathrm{~nm}$

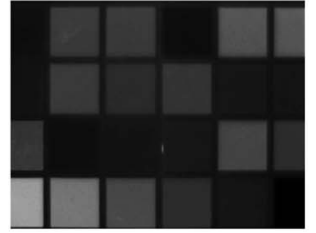

$373 n m$

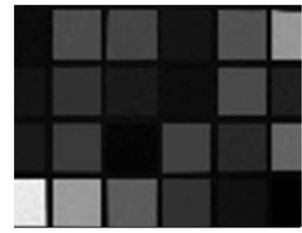

$500 \mathrm{~nm}$

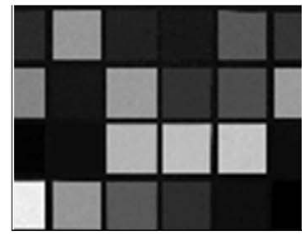

$665 \mathrm{~nm}$

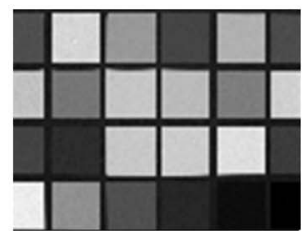

801nm

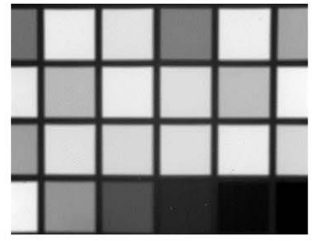

955nm

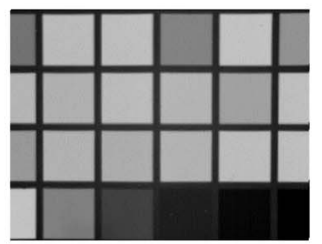

$1451 \mathrm{~nm}$

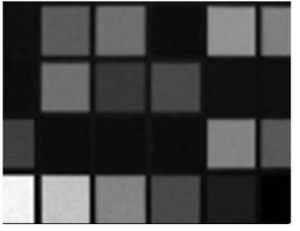

404nm

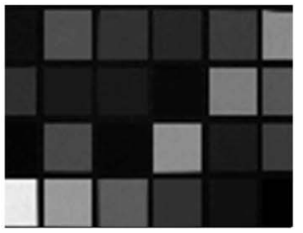

$535 \mathrm{~nm}$

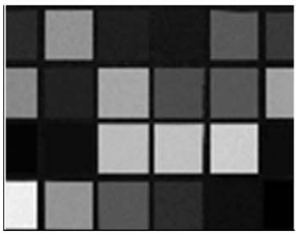

$693 n m$

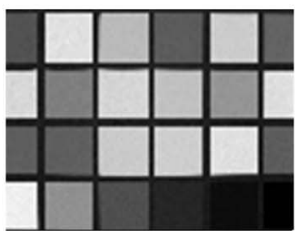

835nm

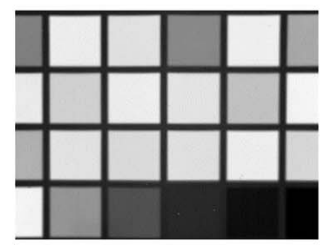

1071 nm

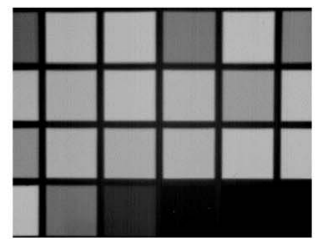

$1540 \mathrm{~nm}$

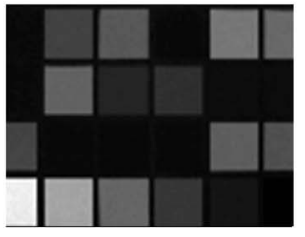

$432 \mathrm{~nm}$

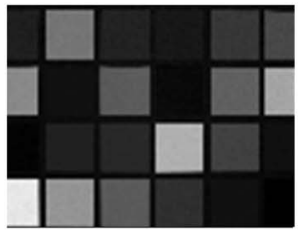

$593 \mathrm{~nm}$

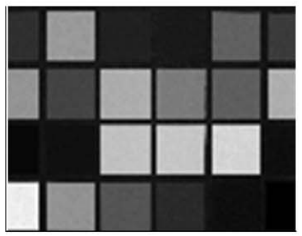

$728 \mathrm{~nm}$

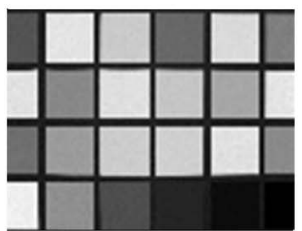

874nm

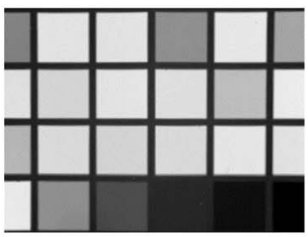

$1202 n m$

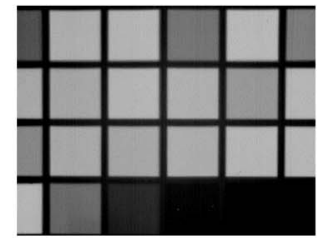

$1630 \mathrm{~nm}$

Fig. 6. RGB image and the 23 spectral images from the CCCR chart obtained with the study system. 
continue to show the system with an excellent performance for this kind of spectral curves. This is shown by the mean values for all the metrics that are close to describing a completely accurate reconstruction.

Table 3 shows the statistical descriptors for the evaluation metrics in the simulations, taking into account the influence of additive random and quantization noise. Additive random and quantization noise reproduce real operational environments, where noise is always present in the output camera responses. These results confirm that the simulations for the pseudoinverse method give better results than the interpolation method, though both achieve adequate results, particularly for module 1 . In module 1 the mean values of RMSE and DE00 were around 1 for the pseudoinverse method, a figure that translates to good performance in colorimetric and spectral reconstruction. The mean values for the

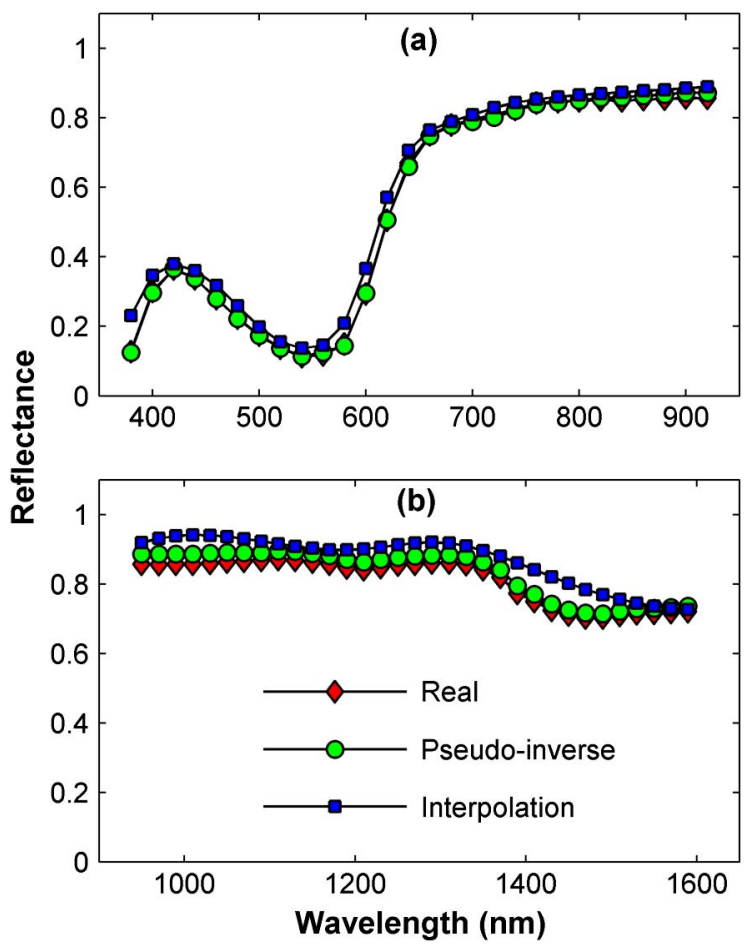

Fig. 7. Spectral reflectances of the cyan color patch in experimental measurements: (a) results for module 1, (b) results for module 2 . Real curves were obtained with commercial scanning spectrometer model Spectro 320 R5 of Instrument Systems.
GFC also support the good spectral performance, since they were above 0.995 . Although the interpolation method showed a poorer performance than the pseudoinverse method, the mean values of DE00 and RMSE of around 2 units are considered adequate. On the other hand, module 2 presented less accuracy in the reconstructions, an expected outcome given the difference in number and spectral width emission of the LED components. In this case, the pseudoinverse method also gave better results.

\section{B. Experimental Results}

After the simulations, real experiments were conducted through the acquisition and processing of data from the patches of the CCCR. Figure 6 shows the 23 spectral images obtained in the measurements and the RGB image formed by means of the spectral channels of 634,535 , and $461 \mathrm{~nm}$. This set of images can be useful by themselves just by direct observation or by using adequate combinations of them and applying pseudocolor rendering to highlight determined features. In our case, because of the differences in sensor formats and spatial resolutions between modules, a digital registering process to combine information from both must be performed. This is a relatively straightforward task, and there are a number of well-established techniques for achieving this $[39,40]$.

Figure 7 shows as an example a graphical comparison of the spectral curves obtained for the cyan color patch, which suggests a similar or even improved behavior to the simulations achieved under influence of additive random and quantization noise (Fig. 5). The results in this figure indicate a better performance in reconstruction for module 1 than module 2 and also indicate that the pseudoinverse method fits more closely with the real spectral curve than the result obtained with the spline interpolation.

Table 4 contains the main statistical descriptors for the evaluation metrics in the experimental measurements of the CCCR chart. The metrics for module 1 with the pseudoinverse method are excellent (mean values of RMSE and DE00 below 0.5), unlike the results of the simulations that included conditions of additive random and quantization noise. The pseudoinverse method also produced very good results in module 2 (mean value of RMSE near 1 and GFC equal to 1.000), better than the corresponding results in the simulations. The results for the spline

Table 4. Evaluation Metrics of Spectral Reconstruction of the CCCR in Real Measurements ${ }^{a}$

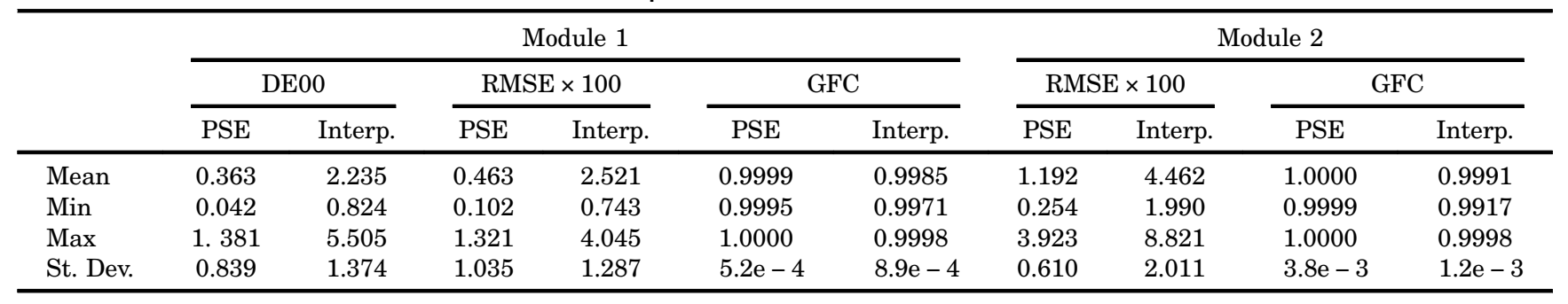

${ }^{a} \mathrm{PSE}$, pseudoinverse; Interp., spline interpolation. 


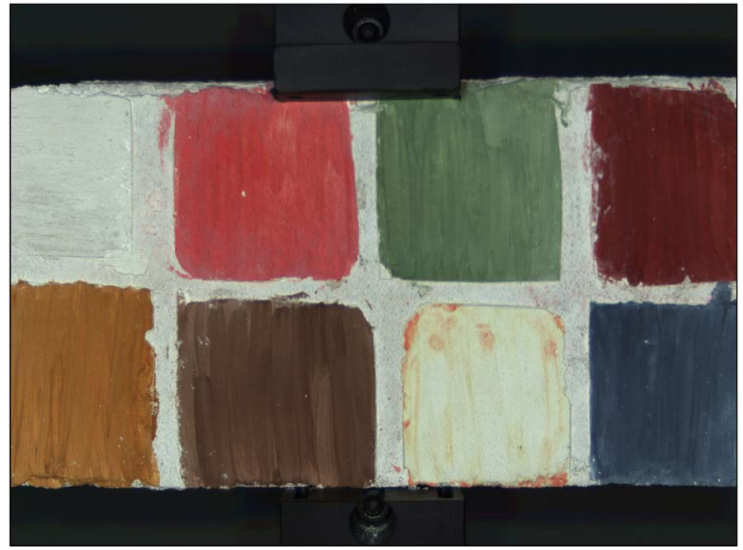

Fig. 8. Palette of pigments built emulating the fresco technique applied in wall paintings.

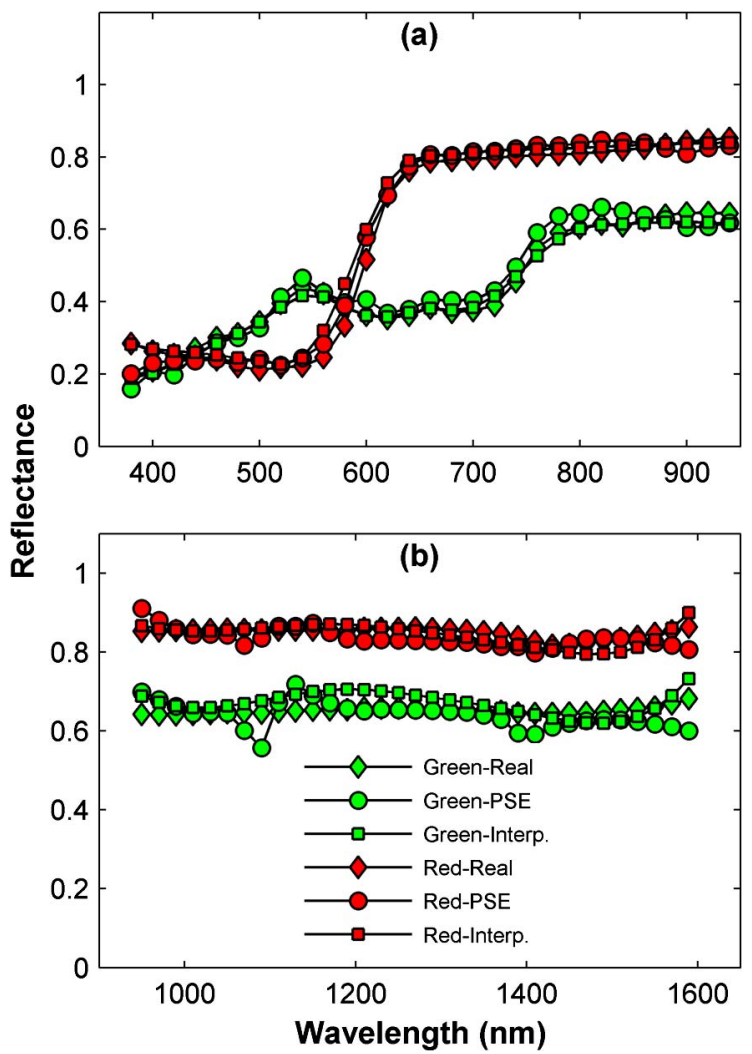

Fig. 9. Spectral reflectance reconstruction for two real samples with green and red pigments using spline interpolation (Interp.) and pseudoinverse (PSE) with the CCCR chart spectral reflectances as the training set. (a) Result for module 1, (b) result for module 2. Real curves were obtained with commercial scanning spectrometer model Spectro 320 R5 of Instrument Systems. interpolation were fairly good, better in module 1 than in module 2, as expected; when comparing the simulations including noise sources, the results for the spline interpolation were very similar.

The difference in improvement between methods from the simulations to the experiments emphasizes the deeper dependence on noise of the pseudoinverse method compared to the spline interpolation technique; indeed, this improvement could have originated from good noise control in the image acquisition by the frame averaging and the time elapsed to achieve stable conditions of illumination while capturing. Therefore, the greater improvement for module 1 with respect to module 2 highlights a difference of control in the thermal sources of noise between cameras: whereas the CCD camera is cooled, the InGaAs camera is not. Thus, the simulation of $2 \%$ noise is accurate for the InGaAs camera of module 2 , but it is probably too high in the case of module 1 .

With the aim of demonstrating the usefulness of the new multispectral system in any kind of sample, we finally obtained spectral images of real samples that did not belong to the classic CCCR chart. Specifically, samples with pigments usually employed in wall paintings were used for this purpose (Fig. 8). To carry out the spectral reconstructions using the pseudoinverse method, the CCCR spectral curves were used as a training set. Figure $\underline{9}$ shows the graphical comparison of these results with the real spectral curves and the spline interpolation reconstructions. The graphical result highlights how the spectral closeness to the training set influenced the accuracy of the spectral reconstructions in the pseudoinverse method. In this case, better metric evaluation values were obtained with the interpolation with splines (Table 5). Nonetheless, an acceptable performance was achieved with the CCCR as the training set, which corroborates the robustness of the system.

The results shown in the experiments and simulations demonstrate that the system performance can be suitable for different applications given the reasonably good performance, and possibilities for modifications as well as evidence its benefits in portability, modularity, and the wide range of spectral acquisition. The system is also attractive as an exploration tool to shed light on the selection of spectral channels and spectral ranges useful in certain specific applications. However, in situations involving the influence of external light sources, dynamic

Table 5. Evaluation Metrics for the Red and Green Samples to test the Spectral Reconstruction Using Pseudoinverse Method with a Different Training Set ${ }^{a}$

\begin{tabular}{|c|c|c|c|c|c|c|c|c|c|c|}
\hline \multirow[b]{3}{*}{ Sample } & \multicolumn{6}{|c|}{ Module 1} & \multicolumn{4}{|c|}{ Module 2} \\
\hline & \multicolumn{2}{|c|}{ DE00 } & \multicolumn{2}{|c|}{$\mathrm{RMSE} \times 100$} & \multicolumn{2}{|c|}{ GFC } & \multicolumn{2}{|c|}{$\mathrm{RMSE} \times 100$} & \multicolumn{2}{|c|}{ GFC } \\
\hline & PSE & Interp. & PSE & Interp. & PSE & Interp. & PSE & Interp. & PSE & Interp. \\
\hline Green & 2.76 & 1.276 & 3.065 & 1.431 & 0.9982 & 0.9996 & 4.544 & 3.901 & 0.9985 & 0.9981 \\
\hline Red & 3.03 & 3.582 & 4.181 & 2.322 & 0.9980 & 0.9986 & 3.667 & 3.100 & 0.9986 & 0.9993 \\
\hline
\end{tabular}

${ }^{a} \mathrm{PSE}$, pseudoinverse; Interp., spline interpolation. 
processes taking place, requirements of high spectral resolution, or large areas that need to be evaluated without further processing and mosaicking, the system might face some of its limitations.

\section{Conclusions}

This study shows the performance of a portable multispectral system based on LEDs covering a wide spectral range. The system comprises two image detectors separated in two modules containing 23 spectral bands in total, built up by LED illumination with varying wavelength emission. The system calibration was carried out using a standard $25 \mathrm{~cm} \times 25 \mathrm{~cm}$ surface white panel of known reflectance. The FOV of the system was adjusted to the aforementioned area, but it can be adapted to meet other specific requirements.

Simulations and experimental measurements using a CCCR chart were carried out to study the performance of the system in spectral reconstruction by employing the pseudoinverse method and the spline interpolation technique for spectral estimation, in conjunction with three different evaluation metrics: DE00, RMSE, and GFC. The simulations included the characteristics of the system and three different conditions of image acquisition: (1) ideal conditions, (2) quantization conditions, and (3) the combination of random additive and quantization noise. These simulations showed good system performance and provided a useful tool to compare and analyze the experimental measurements. The results of the experimental measurements using the spline interpolation method were in agreement with the results of the simulations and demonstrated that the system can be useful even without prior knowledge of the spectral features of the target samples. The results of simulations and experimental measurements using the pseudoinverse method showed differences, which could be explained by two main factors: (1) the control of noise sources had a clear impact on the performance of the pseudoinverse reconstruction method, and the level of additive random noise used in the simulations for module 1 may have been excessive; (2) the use of the same set of samples for the training and test sets may have influenced the results. The measurement of real samples not included in the training set showed that the system demonstrated acceptable performance, with results comparable to those obtained through interpolation. In such cases, better results could be achieved by using a training set similar to the sample to estimate.

In this study, we used LED technology to generate the channels of the multispectral system from the illumination itself, which resulted in an alternative portable, versatile, and cost-effective system for spectral imaging projects. We believe that a wide range of applications can benefit from such systems.

This research was supported by the Spanish Ministry of Science and Innovation under grant DPI2011-30090-C02-01 and the European Union.
J. Herrera thanks the Generalitat of Catalonia for his Ph.D. grant.

\section{References}

1. Q. Weng, ed., Advances in Environmental Remote Sensing: Sensors, Algorithms, and Applications (CRC Press, 2011), p. 610.

2. R. Shrestha, J. Y. Hardeberg, and A. Mansouri, "One-shot multispectral color imaging with a stereo camera," Proc. SPIE 7876, 787609 (2011).

3. N. L. Everdell, I. B. Styles, A. S. Calcagni, J. Gibson, J. C. Hebden, and E. Claridge, "Multispectral imaging of the ocular fundus using light emitting diode illumination," Rev. Sci. Instrum. 81, 093706 (2010).

4. M. B. Bouchard, B. R. Chen, S. Burgess, and E. M. C. Hillman, "Ultra-fast multispectral optical imaging of cortical oxygenation, blood flow, and intracellular calcium dynamics," Opt. Express 17, 15670-15678 (2009).

5. M. Vilaseca, R. Mercadal, J. Pujol, M. Arjona, M. de Lasarte, R. Huertas, M. Melgosa, and F. H. Imai, "Characterization of the human iris spectral reflectance with a multispectral imaging system," Appl. Opt. 47, 5622-5630 (2008).

6. A. Basiri, M. Nabili, S. Mathews, A. Libin, S. Groah, H. J. Noordmans, and J. C. Ramella-Roman, "Use of a multispectral camera in the characterization of skin wounds," Opt. Express 18, 3244-3257 (2010).

7. V. C. Paquit, K. W. Tobin, J. R. Price, and F. Mèriaudeau, "3D and multispectral imaging for subcutaneous veins detection," Opt. Express 17, 11360-11365 (2009).

8. M. Kubik, "Hyperspectral imaging: a new technique for the non-invasive study of artworks," in Physical Techniques in the Study of Art, Archaeology and Cultural Heritage, D. Creagh and D. Bradley, eds. (Elsevier, 2007), Vol. 2, pp. 199-259.

9. R. Padoan, T. A. G. Steemers, M. E. Klein, B. J. Aalderink, and G. de Bruin, "Quantitative hyperspectral imaging of historical documents: technique and applications," in 9th International Conference on NDT of Art (2008), pp. 25-30.

10. E. Marengo, M. Manfredi, O. Zerbinati, E. Robotti, E. Mazzucco, F. Gosetti, G. Bearman, F. France, and P. Shor, "Technique based on LED multispectral imaging and multivariate analysis for monitoring the conservation state of the Dead Sea Scroll," Anal. Chem. 83, 6609-6618 (2011).

11. R. Lu and Y. Peng, "Development of a multispectral imaging prototype for real-time detection of apple fruit firmness," Opt. Eng. 46, 123201 (2007).

12. D. F. Barbin, G. ElMasry, D.-W. Sun, and P. Allen, "Predicting quality and sensory attributes of pork using near-infrared hyperspectral imaging," Anal. Chim. Acta 719, 30-42 (2012).

13. C. Bonifazzi, P. Carcagnì, R. Fontana, M. Greco, M. Mastroianni, M. Materazzi, E. Pampaloni, L. Pezzati, and D. Bencini, "A scanning device for VIS-NIR multispectral imaging of paintings," J. Opt. A Pure Appl. Opt. 10, 064011 (2008).

14. M. Vilaseca, J. Pujol, M. Arjona, and M. de Lasarte, "Multispectral system for reflectance reconstruction in the nearinfrared region," Appl. Opt. 45, 4241-4253 (2006).

15. L. Kong, D. Yi, S. Sprigle, F. Wang, C. Wang, F. Liu, A. Adibi, and R. Tummala, "Single sensor that outputs narrowband multispectral images," J. Biomed. Opt. 15, 010502 (2010).

16. S. Mathews, "Design and fabrication of a low-cost, multispectral imaging system," Appl. Opt. 47, F71-F76 (2008).

17. J. Y. Hardeberg, F. Schmitt, and H. Brettel, "Multispectral color image capture using a liquid crystal tunable filter," Opt. Eng. 41, 2532-2548 (2002).

18. C. D. Tran, "Principles, instrumentation, and applications of infrared multispectral imaging, an overview," Anal.Lett. Part B 38, 735-752 (2005).

19. M. Brydegaard, A. Merdasa, H. Jayaweera, J. Ålebring, and S. Svanberg, "Versatile multispectral microscope based on light emitting diodes," Rev. Sci. Instrum. 82, 123106 (2011).

20. D. Kapsokalyvas, N. Bruscino, D. Alfieri, V. de Giorgi, G. Cannarozzo, R. Cicchi, D. Massi, N. Pimpinelli, and F. S. Pavone, "Spectral morphological analysis of skin lesions with 
a polarization multispectral dermoscope," Opt. Express 21, 4826-4840 (2013).

21. L. Fauch, E. Nippolainen, and A. A. Kamshilin, "Accuracy of the reflectance spectrum recovery in a light-emitting diodebased multispectral imaging system," Opt. Eng. 51, 053201 (2012).

22. J. Li and R. K. Y. Chan, "Toward a UV-visible-near-infrared hyperspectral imaging platform for fast multiplex reflection spectroscopy," Opt. Lett. 35, 3330-3332 (2010).

23. J. K. Delaney, J. G. Zeibel, M. Thoury, R. Littleton, M. Palmer, K. M. Morales, E. R. de la Rie, and A. Hoenigswald, "Visible and infrared imaging spectroscopy of Picasso's Harlequin musician: mapping and identification of artist materials in situ," Appl. Spectrosc. 64, 584-594 (2010).

24. H.-L. Shen, J. H. Xin, and S.-J. Shao, "Improved reflectance reconstruction for multispectral imaging by combining different techniques," Opt. Express 15, 5531-5536 (2007).

25. H. Andrews, "Cubic splines for image interpolation and digital filtering," IEEE Trans. Acoust. 26, 508-517 (1978).

26. J. Kiusalaas, Numerical Methods in Engineering with MAT$L A B$ (Cambridge University, 2005).

27. M. Melgosa, A. Trémeau, and G. Cui, "Colour difference evaluation," in Advanced Color Image Processing and Analysis, C. Fernandez-Maloigne, ed. (Springer, 2013), pp. 65-85.

28. G. Sharma, W. Wu, and E. N. Dalal, "The CIEDE2000 colordifference formula: implementation notes, supplementary test data, and mathematical observations," Color Res. Appl. 30, 21-30 (2005).

29. M. R. Luo, G. Cui, and B. Rigg, "The development of the CIE 2000 colour-difference formula: CIEDE2000," Color Res. Appl. 26, 340-350 (2001)

30. J. Hernández-Andrés, J. Romero, and R. L. Lee, "Colorimetric and spectroradiometric characteristics of narrow-field-of-view clear skylight in Granada, Spain.," J. Opt. Soc. Am. A 18, 412-420 (2001).

31. F. H. Imai, M. R. Rosen, and R. S. Berns, "Comparative study of metrics for spectral match quality," in Proceedings of the First European Conference on Colour in Graphics, Imaging and Vision (2002), pp. 492-496.

32. A. Kimachi, H. Ikuta, Y. Fujiwara, M. Masumoto, and H. Matsuyama, "Spectral matching imager using amplitudemodulation-coded multispectral light-emitting diode illumination," Opt. Eng. 43, 975-985 (2004).

33. T. Nägele, "White light LEDs-importance of accepted measurement standards," LED Prof. Rev. (10) 22-26 (2008).

34. Ó. Martínez, M. Vilaseca, M. Arjona, C. Pizarro, and J. Pujol, "Use of light-emitting diodes in multispectral systems design: variability of spectral power distribution according to angle and time of usage," J. Imaging Sci. Technol. 55, 050501 (2011).

35. M. de Lasarte, J. Pujol, M. Arjona, and M. Vilaseca, "Optimized algorithm for the spatial nonuniformity correction of an imaging system based on a charge-coupled device color camera," Appl. Opt. 46, 167-174 (2007).

36. N. Shimano, K. Terai, and M. Hironaga, "Recovery of spectral reflectances of objects being imaged by multispectral cameras," J. Opt. Soc. Am. A 24, 3211-3219 (2007).

37. R. Shrestha, A. Mansouri, and J. Y. Hardeberg, "Multispectral imaging using a stereo camera: concept, design and assessment," EURASIP J. Adv. Signal Process. 2011, 57-71 (2011).

38. K. Barnard and B. Funt, "Camera characterization for color research," Color Res. Appl. 27, 152-163 (2002).

39. J. Sarvaiya, S. Patnaik, and K. Kothari, "Image registration using log polar transform and phase correlation to recover higher scale," J. Pattern Recogn. Res. 7, 90-105 (2012).

40. R. C. Hardie, M. M. Hayat, E. Armstrong, and B. Yasuda, "Scene-based nonuniformity correction with video sequences and registration,” Appl. Opt. 39, 1241-1250 (2000). 